\title{
La urbanización crítica en la metrópoli de São Paulo, a partir de fundamentos de la geografía urbana ${ }^{1}$
}

\author{
Amélia Luisa Damiani²
}

\begin{abstract}
RESUMEN
A lo largo del tiempo la ciudad se ve envuelta en constantes cambios propios de su diversidad, procesos y estructura interna. Al igual que la realidad urbana, los conceptos de la geografía urbana como disciplina presentan diversas metamorfosis basadas en la agitación de dicha cambiante realidad. Esta investigación pretende acercar a una actualización de la bibliografía geográfica, sin simplificaciones extremas e incorporando todas sus perspectivas posibles. En este contexto es que dentro de la bibliografía existente, destaca la revisión de un texto de Pierre George, quien entrega un ejemplo extraordinario de la extensión de ese conocimiento, precipitadamente convertido en obsoleto.
\end{abstract}

Palabras clave: Concepciones de la geografía urbana, urbanización empírica, urbanización crítica.

\begin{abstract}
Over time the city has been involved in constant changes related with its diversity, processes and internal structure. As urban reality, the concepts of urban geography as a discipline shows a metamorphosis based on the agitation of urban reality. This investigation aims to update the geographical literature, without extreme simplifications and including as much perspectives as possible. Pierre George gives and extraordinary example about the extension of that knowledge, abruptly become obsolete.
\end{abstract}

Key words: Concepts of urban geography, empirical urbanization, critical urbanization.

Es extraordinario examinar el libro de Pierre George (1983), Geografía urbana, cuya primera edición es de 1961 a la luz de la realidad de la urbanización de nuestros tiempos. Se trata de encontrar la potencia en la observación de los fenómenos urbanos, en los años 1950-1960, y evaluar cómo el cuerpo conceptual de la geografía lidiaba, en ese período, con fenómenos tan mutables como los que se refieren a lo urbano.

1 Artículo recibido el 5 de junio de 2009 y aceptado el 5 de marzo de 2010.
El autor buscó en la geografía una concepción de totalidad de los procesos geográficos, que atravesaban las ciudades en el mundo, en los años 1960. El libro data como se señaló de 1961. No obstante, ya en 1952 George escribió sobre el tema, desde ese punto de vista: el de una geografía general sobre lo urbano en el mundo, sugiriendo tipos de ciudades y los condicionantes de su

\footnotetext{
2 Departamento de Geografia, Universidad de São Paulo (Brasil). E-mail: ameluisa@usp.br
} 
crecimiento urbano, de su repartición desigual en el mundo, en estudios comparativos (George, 1952; George, 1983).

Los instrumentos analíticos en boga en la época, conceptualizaban a la ciudad como un organismo urbano. Esa concepción orgánica atraviesa la geografía alemana y francesa, resbala en la influencia de las ciencias naturales y biológicas en el interior de las ciencias humanas, lo que fue conceptualizado como positivismo en la geografía, y es posible incluirla en los fundamentos del pensamiento urbano de Pierre George. Pero tales instrumentos analíticos ya presentaban señales evidentes de su ruptura y límite. En George (1983), las ciudades son conceptualizadas como organismos urbanos, y se agrega a la conceptualización la idea de aglomeración urbana: la ciudad y sus aglomeraciones urbanas, al final, se sintetizan las nociones de fundamento. En rigor, esa composición ya es contradictoria y sostiene una situación de impasse, inclusive conceptual: la negación de la ciudad como organismo urbano coherente y solidario en sus elementos constitutivos. La noción de aglomeración urbana expresa la inminente contradicción. Lecturas recientes del autor demuestran la potencia crítica de sus escritos sobre lo urbano, vislumbrando la crisis urbana.

Se puede establecer la siguiente tesis: el libro (George, 1983) presenta un descompás entre las observaciones empíricas del proceso de urbanización y el acervo conceptual disponible; al mismo tiempo, de modo rico y complejo, anuncia nuevas concepciones, confirmadas por la realidad urbana actual. La interpretación del libro sugerido, por lo tanto, es informada por las nuevas aproximaciones y fenómenos urbanos. Su lectura compuso su primera y segunda parte simultáneamente, ya que la segunda elucida conceptualmente el trayecto empírico de la primera.

\section{De la urbanización difusa a la urbanización empírica}

En una línea de continuidad, que intenta descifrar el proceso de urbanización, como es pensado por Pierre George, a la luz de la primera parte de la obra denominada formación del marco urbano, se tiene la siguiente evolución: de la urbanización difusa, de creci- miento gradual y constante, a la urbanización empírica, de crecimiento abrupto y acelerado.

En el transcurso del texto, la concepción recibe más plasticidad, absorbiendo el tratamiento del fenómeno urbano en su complejidad, aunque, conceptualmente, aún hay hiatos: la ciudad y sus aglomeraciones urbanas - la ciudad no viene sola, sino siempre acompañada de ese agregado designado como "aglomeraciones urbanas", formando lo que el autor designa como conjunto urbano-.

Aunque el tratamiento de las ciudades incluya los barrios, como modo de organización del espacio, aparecen los términos analíticos de la urbanización empírica, contraria a esa organicidad, en principio designadora del fenómeno: zonas periféricas, áreas suburbanas, guetos, segregación urbana, hábitats espontáneos, como áreas incluyendo poblaciones no integradas. Las peculiaridades de los Ilamados países subdesarrollados serían reveladoras de esa realidad inestable.

El fenómeno urbano es tratado en extensión: varios modos de crecimiento, cuyo tenor mayor es la aceleración del proceso de concentración de población en las ciudades, que, a su vez, define cambios intensivos de ellas. Las metamorfosis socioculturales de lo urbano equivalen a las pérdidas de los modos de vida, propios de colectividades singulares, de identidades perennes, y a la afirmación de identidades regionales y nacionales provisorias, como el caso de guetos; el sentido es el del agotamiento de esas identidades concretas.

La perspectiva de la ciudad como organismo urbano es también la comprensión de la ciudad como obra humana, aquella que refleja y, al mismo tiempo, humaniza al hombre. En esta literatura geográfica sobre la ciudad se prevé un embate sordo, no reflejado completamente, ambiguo teóricamente, entre la humanización y la deshumanización, expresado en la mantención de la idea de la ciudad como organismo urbano. Esa ambigüedad conceptual también es la de la relación no plenamente resuelta entre lo inmediato y lo mediato; la dificultad de la comprensión de la presencia constitutiva de la abstracción en las relaciones sociales modernas. Por otro lado, el espacio guarda otros tiempos, incluyendo los de la inmediatez de las relaciones 
sociales. Los propios principios de tratamiento de la población, por edad y sexo, equivaliendo a diferencias primarias, aún mantiene ese carácter antropológico.

Pero la voracidad del proceso urbano, de urbanización, recibe, en ese método geográfico, un impulso necesario y considerable, inclusive, poniendo de alguna manera en cuestión, el concepto de organismo urbano, en el caso del estudio de las ciudades. Se estaría pasando hacia algo relativo a la noción de medida -cuando la cantidad deja de ser estrictamente un elemento indiferente y pasa a ser activo, impulsando la dialéctica entre cantidad y calidad-(Hegel, 1995).

El reconocimiento es el de la universalidad y el de la aceleración del crecimiento de los grandes aglomerados urbanos (de millares a millones de habitantes); este sugeriría tipos de ciudades. Se vislumbra la necesidad de comparación de las ciudades en el mundo. Se mide la extensión y la intensidad de la ocupación territorial urbana que, al mismo tiempo, sugiere un tratamiento en espectro, de situaciones globales a escalas intraurbanas, considerando las transformaciones del aspecto físico urbano y de la vida cotidiana en lo urbano, por ejemplo, el ritmo de crecimiento de las periferias, con superpoblación crítica (densidad mayor de ocupación por habitación del hogar; afluencia mayor de inmigrantes hombres jóvenes y parejas jóvenes; hasta zonas suburbanas, en tanto que consumidoras de hombres, "pues en ellas la muerte sobrepuja a la vida", cuando el empleo es inestable). La corona suburbana se presenta como frente pionero y de colonización de la urbanización. Esa lectura manifiesta su actualidad incontestable. En lo urbano se reproduce el aspecto crítico de la economía capitalista: en el límite, "el enorme contraste de renta entre clases dirigentes y propietarias y la masa de la población sin poder adquisitivo, que vive, en el sentido propio del término, el día a día" (George, 1983: 165).

Por un lado, la ciudad configura una unidad demográfica, por otro, las colectividades geográficas -inclusive los barrios- son sustituidos por las relaciones socioprofesionales. La superación de la tradición de pensar el medio geográfico y sus diferenciaciones a través de modos de vida -de la relación entre el hombre y la naturaleza y entre los hombres como un proceso de apropiación social de la naturaleza, de larga duración-, con la participación de la noción de división social de trabajo, en el centro del debate sobre el desarrollo de las actividades productivas, y su organización espacial, significó un importante cambio en la literatura geográfica y en nuevas relaciones con la sociología, aunque conservadores, pues prevalecería una comprensión progresista del capitalismo, de reconocimiento de las abstracciones concretas, propias del mundo moderno; de la realidad de las relaciones sociales formales, sintetizadas en la alienación social del trabajo. Los desdoblamientos teórico-metodológicos para la geografía llevan a la centralidad, hoy, de la categoría de producción del espacio.

El trayecto del conocimiento del objeto de estudio de la geografía urbana, que se anuncia a través de los estudios poblacionales y se desdobla en la repartición geográfica de las ciudades en el mundo, con sus cualidades socioculturales, muchas veces de raíces de larga duración, definiendo una regionalización del fenómeno en el mundo; ese trayecto, comprendido en el plano más amplio del proceso de conocimiento en general, tiene la profundidad del desarrollo del conocimiento moderno, así como sus impasses: la relación dialéctica entre lo infinito y lo finito; entre la cantidad y la calidad; entre el análisis y la síntesis; entre la parte y el todo, la superficie terrestre como un todo y la particularidad, inclusive la individualidad, de los lugares ${ }^{3}$. A continuación se enuncian esas derivaciones, aunque esbozadas, a grandes rasgos.

3 El objeto de la geografía, estudiando la relación entre el hombre y la naturaleza y entre los hombres, contiene la infinitud de lo que es esencial de la naturaleza; de la relación entre el hombre y la naturaleza; y entre los hombres; y de sus relaciones mutuas. Pero al mismo tiempo, se debe realizar como finitud y lo hace eligiendo la superficie de la tierra como totalidad. Siempre recordando la plasticidad de esa base material y finita: el ecúmeno, en constante aumento; inclusive con el desarrollo científico y técnico. Hay una contradicción dialéctica implicada en el objeto: la relación entre infinito y finito. Lo importante a ser considerado es que la contradicción existe pero puede no estar explicitada, pensada, resuelta, en tanto que dialéctica, por la geografía. 
En el transcurso del libro, que sirve de base y ejemplo, la Geografía urbana de Pierre George (1983), después de esa aproximación del fenómeno urbano, a través de la población y sus índices, el autor discurre sobre la repartición de las ciudades en el mundo. Lo importante a considerar, metodológicamente, es la contradicción dialéctica cantidad-calidad puesta en ese desarrollo. Contradicción que puede resolverse en el libro, a nivel de sus conceptos más evidentes, de modo formal, pero que es latente y de profundo resultado en la definición del trayecto de desvendamiento del objeto de estudio.

Si en los primeros capítulos de las dos partes del libro, la noción de cantidad funda la inteligibilidad del recorrido, con la idea del desciframiento de los datos poblacionales, los capítulos sobre la regionalización del fenómeno urbano traen una base cualitativa que subvierte lo anterior. Es la densidad de los modos de vida, de las culturas, de las técnicas y del trabajo humano, traducida en las configuraciones geográficas. La naturaleza del análisis altera sus procedimientos. Los documentos históricos pasan a ser parte necesaria del acervo de consulta. Y la tragedia de los cambios urbanos y sus desigualdades se va exponiendo.

Aquí, un elemento importante en el texto es la introducción de los procesos económicos modernos y las configuraciones geográficas, resultantes y presupuestos de los mismos -causa y efecto, en su relación mutua-. Así, las reparticiones geográficas llegan a la regionalización de los países desarrollados y subdesarrollados, como momentos necesarios del moderno proceso de desarrollo económico.

Un momento demarcador del libro base de este ensayo, referente a la dialéctica entre cantidad y calidad en la geografía urbana, es la idea del proceso de urbanización que, Ilevado al límite, incluye la noción de urbanización empírica, de extraordinaria posibilidad, porque anuncia, en esa obra, la implosiónexplosión de la ciudad como obra humana (Lefebvre, 1970; Lefebvre, 1974).

Así, el eje de la comprensión del fenómeno urbano, como movimiento de la práctica social - práctica social que se podría calificar como alienada, inclusive desde la perspectiva del texto en análisis-, tiene el proceso de urbanización como base.

La base filosófica de la repartición geográfica se asienta en la enorme conquista de la filosofía moderna que es la comprensión, la destrucción del objeto para constitución de su inteligibilidad (Lefebvre, 1947). Participa de la negación de la conquista de la unidad, de la totalidad como premisa metafísica escolástica o mágica. La repartición geográfica de las ciudades en el mundo, en este caso, comporta una tradición de geografía comparada ${ }^{4}$, propia de su tenor disciplinar, lo que significa decir que a la repartición se agregan las nociones de encadenamiento, de relación de conjunto; los fenómenos particulares subordinándose al ámbito de la tierra tomada en su conjunto.

Esa geografía comparada tiene como principio una geografía general en la relación con las particularidades, hasta singularidades, de los lugares. Las monografías urbanas se componen con estudios generales y comparativos.

\section{La noción de espacio}

En este libro, Pierre George (1983), aunque de modo no pensado plena y conceptualmente, ejercita la metamorfosis del espacio absoluto en espacio relativo. Se divide en esas dos pertenencias de la geografía: la tradicional, antropológica, y la contemporánea, que debe contener una concepción social de la historicidad y la espacialidad moderna.

La noción de espacio absoluto, definido de modo materialista, confirma la no problematización de la relación partes y todo; cada cual expresando el otro sin fisuras. De esa manera, la sociedad moderna, basada en la negación entre sujeto y predicado (en términos más abstractos), no es suficiente y crucialmente expresada en sus contradicciones. Se define así el plano mental, y al mismo tiempo, social, del espacio absoluto. Mentalmente es el espacio geométrico y euclidiano; socialmente es la sociedad moderna, el presente, sin contradicciones reflejadas como tales; las que por lo menos los fundamentos lógicos

\footnotetext{
4 Se reservaría a la geografía el tratamiento de las diferencias localizadas en la superficie de la tierra, comparándolas (Capel, 1981).
} 
del pensamiento geográfico lo aclaran. Pero es exactamente Pierre George y su grupo que, a mediados de los años 1960, definen la idea de espacio relativizado. El propio espacio como situación (George et al., 1966).

El debate sobre el espacio, definido como absoluto, que aparece como fundamento lógico de las concepciones en curso, al mismo tiempo, ofrece la oportunidad de actualizar el libro y ponerlo en relación con las geografías posmodernas, de Edward Soja (1993), Ilevando ambos a un recorrido por el pensamiento en geografía urbana.

Como espacio absoluto, todo se propone como elemento de la organización del espacio. Nada se separa de modo estricto, o sea, nada es simplemente decorativo. La organización aquí recibe el sentido del obedecer a una orden. No existe lo fortuito. La noción de superficie de la tierra prevalecerá y equivale a componer el mundo y su repartición, excluyendo el arriba y el abajo: el cosmos y las profundidades, a no ser cuando establecen relaciones evidentes con esa superficie, cuando técnica y socialmente pasan a formar parte de la organización del espacio social. A las alturas, se reserva la ciencia de la astronomía, y a las profundidades, la psicología e inclusive la sociología. Pero las condiciones climáticas son parte de la superficie; así como la geomorfología absorbe la geología; y ambas pueden configurar condiciones naturales a partir de las cuales el hombre actúa y define segmentos de la geografía como ciencia. La naturaleza humanizada, la producción de calidades socioculturales y sus diferencias construyen la geografía humana, o, específicamente, la geografía urbana, como civilizatoria. La base es la naturaleza y la naturaleza modificada; el principio de la propiedad privada, en sentido lato, que se afirma como trazo irrevocable del proceso de modernización, va a aparecer a veces como residual; una vez establecido, se llega a la noción de espacio relativo y no absoluto: involucrando mediaciones abstractas, definidoras del espacio de la acumulación. En la repartición geográfica de las ciudades en el mundo, en su regionalización, hay un lugar prominente para las cualidades socioculturales de los espacios; la realidad de las abstracciones concretas, económicas, va apareciendo a posteriori, no es principio formador del argumento.
La argumentación aquí desarrollada sobre la noción de espacio absoluto, en el sentido concreto, materialista del término, como fundamento de una geografía urbana, no equivale a descaracterizarla como anunciadora de lo que se tornó la producción del espacio -incluyendo el espacio como relacional ${ }^{5}-$, sino que se trata de demostrar un movimiento del conocimiento que es también, a partir del reconocimiento de las diferencias y prácticas socioespaciales, el de la superación, más o menos consciente, de la naturaleza como base y principio, como superficie de la tierra, en beneficio del análisis de la naturaleza abstracta -la segunda naturaleza, producida por el hombre y alienada de él-, exigida como fundamento de lo moderno. En el plano fenomenológico puede estar presente, en la argumentación, el sentido último de la modernidad, pero los instrumentos lógicos adecuados a su conocimiento, aun tienen que ser configurados. Es decir, al inicio hay un proceso homogeneizador, en relación y destructivo de las particularidades, y no las propias particularidades regionales $y$ locales a descifrar, después del reconocimiento de esa abstracción concreta.

\section{El trabajo de Pierre George anuncia una geografía crítica}

El segundo capítulo del libro en examen es sobre el origen y la génesis de las ciudades; por lo tanto de sus particularidades. En él, el autor identifica diferentes generaciones de ciudades en el mundo, convergiendo, sin embargo, para tipos de ciudades. Los tipos definen los modernos procesos económicos y sociales, que atraviesan las ciudades, con historias y pasados diferentes y de modo distinto. Una regionalización, como dice, en sentido amplio, es el plano analítico; o sea, el conjunto urbano en/de los países; las funciones económicas de las ciudades, definiéndolas; y la distribución de las funciones en el interior de cada ciudad. La composición de este capítulo con el segundo capítulo de la segunda parte del libro, sobre trabajo urbano, ayuda a examinar su actualidad conceptual.

\footnotetext{
5 “Debemos, también, pensar relacionalmente el espacio porque hay un sentido importante en el cual un punto en el espacio 'contiene' a todos los otros puntos [...] y es también crucial para entender la determinación del valor del suelo..." (Harvey, 1980: 143-144).
} 
Una ruptura conceptual, histórica, que mueve la transformación de las ciudades, representa el continuo de todo el análisis: la industrialización; siendo que esta y sus derivados, entendida como la extensión de los servicios y el comercio -más o menos contradictorios-, implican formas de trabajo concentrado, en fenómenos de masa, que metamorfosean las ciudades.

El desarrollo posterior de las ideas así lo recomendará: reflexionar acerca de la ciudad como región urbana y como centralidad en torno a una periferia; o a una complejidad de centralidades y periferias.

En el interior de un raciocinio aparentemente desarrollista, el autor formula una tesis crítica sobre la evolución de las ciudades: el crecimiento de las ciudades conduce a la negación (imposibilidad) del urbanismo (como proyecto colectivo, social) y está bajo el privilegio de la economía (industria, finanzas, circulación y especulación) y de la arquitectura (como solución individual, aislada).

Las ciudades, como fenómenos de larga duración, remiten a un plano histórico continuo, a partir del cual se localiza una discontinuidad, que redefine ese complejo espaciotemporal ${ }^{6}$.

Con instrumentos conceptuales aún ambiguos, preservando una idea de organicidad de la ciudad, el autor anuncia, en un movimiento lógicamente no explicitado como contradictorio: los límites de lo urbano en la sociedad capitalista moderna. Para fundamentar la actualidad del autor, se puede

\footnotetext{
6 Algunas tentativas para demostrar la extensión e intensidad de los cambios son expresadas de la siguiente forma:

- Sobre la revolución industrial en lo urbano, en el ámbito planetario se tiene: ciudad nueva (creación de la industrialización); ciudad con pasado, transformada (por la industrialización); y ciudad tradicional (ecos de la transformación);

- Como características del movimiento urbano: sociedad rural (ciudad y conjunto patriarcal); coexistencia de arcaísmos y modernismos (dos ciudades); y sociedad urbana (separación entre las actividades productivas y las actividades residenciales, incluyendo la utopía de los pabellones y los movimientos diarios de la población activa), sumando la idea de conurbaciones industriales.
}

desviar de algunas maneras, como se intenta hacer en este trabajo. Será preciso osar más, en el desvío, a continuación. Siendo el desvío la práctica de utilizar al autor de manera irreverente, no para vulgarizarlo o enaltecerlo, sino para situarlo en otro nivel de relación, en este caso, con el conocimiento geográfico por venir ${ }^{7}$.

La composición desviada entre los terceros capítulos, de las partes 1 y 2 del libro en examen, permite un tratamiento inusitado: a partir de las condiciones naturales, que arraigan las ciudades, cuyo elemento analítico más evidente es el sitio urbano, en escala local, para circunscribir esta escala, superándola, en la relación con otras escalas, como la regional. Así, el par paradigmático de los estudios urbanos en geografía se remite al sitio y a la situación de la ciudad; par que propone la concepción de lo urbano como relativa a diferentes escalas: en el tiempo y en el espacio. En el tiempo, el sitio se va desactualizando, las condiciones naturales van perdiendo valor explicativo en la morfología de la ciudad; no obstante, el análisis remite a los inicios de la formación del núcleo urbano, en una escala espacial local y a la historia de su desarrollo morfológico. La situación define un segmento regional de tratamiento de la ciudad, su proceso de irradiación, y garantiza, todo el tiempo, el examen de la actualidad de las condiciones materiales de relación de la ciudad con su entorno; las condiciones de desarrollo urbano, en el ámbito de la organización de los medios de transporte, de la circulación e intercambio.

En este sentido, el problema fundamental de la geografía urbana es el problema de la geografía de la circulación, abarcando el desplazamiento de hombres y mercaderías en cantidades de crecimiento exponencial. La geografía se reitera por su naturaleza de fenómeno de masa. Bajo impulsos naturales particulares, la posición de la ciudad recibe a los medios de comunicación siempre renovados y aumentados. La concepción, entonces, en la base de esas transformaciones, es la formación de un sistema de organización territorial como factor decisivo. La escala geográfica es la regional, en sentido amplio, inclusive,

\footnotetext{
7 Sobre la noción de desvío ver Mosconi, 1997.
} 
en cuanto a la situación y en la escala temporal cuenta la actualidad de las conquistas técnicas y científicas, que mueve las nuevas condiciones materiales de circulación en general. Esta geografía, que se va forjando, en lo urbano, es la de la circulación, inclusive del punto de vista de la vida cotidiana: la noción de ambiente equivale a concebir cómo las formas de organización de la vida material interfieren en la vida cotidiana. La circulación necesaria de grandes masas de población a diario. La movilidad de la población urbana localiza un temario apreciado por los estudiosos de lo cotidiano, el que aparece en esa obra: el tiempo obligatorio, la imposición (o la imposibilidad) de una penosa circulación diaria.

Del par sitio y situación no se extrajo solamente un darwinismo de la formulación en geografía urbana, sino también la actualidad de su tratamiento de la cotidianidad (cuya formulación teórica apenas esboza). Para una lectura más vulgarizada, la estructura analítica de apoyo de este libro, en este momento, reiterada en compendios de geografía urbana, hace prevalecer la arquitectura de los condicionamientos naturales.

No obstante, se tiene como movimiento de superación de los condicionamientos naturales un sistema de organización territorial y ambiente y vida cotidiana.

A través del transcurso (expuesto anteriormente), el sitio y la situación que va desde condicionantes naturales al sistema territorial organizado, pasando por el ambiente suburbano, a la vida cotidiana de los citadinos, como modo de deterioro de la vida urbana, se tiene un trayecto anunciado que no cabe en aclaraciones sobre si es positivista o no el argumento del autor, pero que el mismo, con instrumentos conceptuales insuficientes, Ilega a considerar la alienación social a través de lo urbano.

En el capítulo tercero, "Aspectos de la vida social urbana", de la segunda parte del libro Geografía urbana, Pierre George (1983) anuncia, sintética y conceptualmente, la tesis del texto, de la cual derivará su estructura analítica en fases de la vida en la y de la ciudad: movilidad cotidiana de la población, acceso a los equipamientos comerciales, a los servicios de todos tipos, búsqueda de la recreación y el descanso; lo que, en la condición de fenómeno de masa, comporta situaciones de vida provisorias e inestables. Esta vulnerabilidad social es propia de la condición citadina en sociedades industriales.

El compuesto actividades productivas - movidas por el proceso industrial- y vida cotidiana en la ciudad es explosivo, redefiniendo los límites del fenómeno urbano. Las formas de irradiación para más allá de la ciudad llevan a la consideración de las redes urbanas, de las formas de conurbación, de la constitución de periferias. El cuadro tradicional de la ciudad se rompe, y ella es, al nivel de la vida cotidiana, penosamente soportada. "Este esquema general debe ser retocado en función del desigual desarrollo económico y social de los diversos países y también de ciertas modalidades de la organización de la vida colectiva" (George, 1983: 203).

El plano del análisis de los últimos capítulos (cuarto y quinto de la primera parte y último de la segunda), sintéticamente, puede ser reconducido a partir de la idea de espacio de catástrofes (Oseki, 1956).

La organización del espacio urbano, redefinida y cada vez más funcional, insertada en la dinámica abstracta de la economía moderna, que materialmente se transforma de manera exponencial ${ }^{8}$, puede comportar el análisis de los puntos de ruptura, de los puntos críticos de la estructuración en curso. El argumento no fluye solamente en el sentido de la coherencia y del equilibrio de las nuevas realidades urbanas, sino que contiene contradicciones no pensadas como tales, expuestas como realidades sociales a corregir, en el ámbito colectivo estatista. El sentido de esa realidad urbana, como elemento crucial de las contradicciones modernas, no fue totalmente tratado, pero el texto incluye momentos que crean esa posibilidad.

8 Es, inclusive, bello y dramático el texto sobre Tunis, ciudad colonizada por los franceses, representativa de lo que vino a ser otras ciudades del norte de África (George, 1983). 
De la urbanización difusa a la empírica, existe la exposición del espacio de catástrofe del organismo urbano, como forma de agrupamiento y de actividad: la explosión de las ciudades en periferias en tanto unidades urbanas incompletas. La realidad es el aglomerado urbano. La ciudad no es sino una fracción, el fenómeno puede ser interpretado como "una bola de nieve"; bajo la presión de los negocios financieros, ocurre expansión, con la expulsión de la mayor parte de la población de los núcleos citadinos y metropolitanos centrales.

¿Cuál es el paso de esa geografía que se ha explorado de alguna forma a aquella que se tornó la geografía contemporánea crítica?, ¿qué acervó las diferencias?, ¿cómo?, ¿profundamente?, ¿de manera superficial? A nivel de la historia del pensamiento geográfico, hubo una ruptura nítida entre lo que fue la geografía y en lo que se transformó, la geografía crítica o, para los angloamericanos, la geografía radical (Johnston, 1986).

Aquí se indica la dificultad de demarcar la ruptura, como discontinuidad absoluta. El movimiento de superación de la geografía anterior en relación a la geografía contemporánea crítica está en su fundamento marxista: la crítica a la economía política.

El texto Geografías posmodernas... (Soja, 1993), discutiendo la dialéctica del espacio, de modo más abstracto, localizando su sentido en la literatura marxista (su punto de ruptura con la dialéctica del tiempo), la dialéctica del tiempo monopolizando esa misma literatura, al descifrar los fundamentos del capitalismo y, sintetizando el argumento, a través de un ensayo paradigmático sobre Los Ángeles, pudo sugerir como ejercicio metodológico, otros instrumentos conceptuales y formales de interpretación geográfica; una discontinuidad del pensamiento en geografía urbana. Ahora, esa discontinuidad puede presentarse, con este y otros autores contemporáneos, sin forjarse en la negación estricta de lo que fue antes la geografía urbana.

La geografía que se ha discutido elucida la grandeza del proceso social de modernización, que alcanza a todos, aun en los lugares más distantes de su centralidad; aunque tal proceso sea, inherentemente, desigual. Inclu- sive, la expresión descriptiva de esa presencia señala la metamorfosis o dura destrucción de tradiciones y culturas pasadas. Una geografía general, que comportaba la diversidad del fenómeno geográfico del mundo, se definió como momento necesario del proyecto de geografía humana; en este caso tratado, realizándose como geografía urbana.

Se anuncia el capitalismo financiero: incluyendo la descripción de emprendimientos económicos y especulativos involucrados, barriendo las historias de pueblos colonizados, reproduciéndose en imperios y constituyendo una masa de población potencialmente trabajadora, agigantada y concentrada, especialmente, en grandes ciudades perversamente concentradas, pues se denuncian las formas de segregación espacial producidas.

El pensamiento marxista, cada vez más interfiriendo en la geografía producida, acabó por causar una nueva hipótesis teórico-metodológica para la geografía, aún en constitución: la geografía deja de ser periférica en el interior de una teoría social crítica, y pasa a ser constitutiva de ella.

En la obra de Soja (1993) aparece la idea de la importancia de la interpretación marxista del mundo para el desarrollo geográfico desigual. Él tendría el estatuto de las contradicciones de clase en la explicación del capitalismo, de la relación capital-trabajo. Por ese camino, el autor llega a la necesidad de una concepción dialéctica del espacio. En esta obra es posible comprender la centralidad del pensamiento de Henri Lefebvre, que habría participado de una unión de tendencias que se afirmaban dentro y alrededor del marxismo, con cierta dificultad, con el propósito de una dialéctica espacial y encontrar su sentido en la teoría social crítica. Del punto de vista abstracto-conceptual, la noción de heterotopías, de Foucault, ponía otro registro espacial en la interpretación del mundo moderno, aunque el autor, también, la haya identificado como constitutiva de otras sociedades, a lo largo del tiempo histórico. "La heterotopía es capaz de superar en un único lugar real, diversos espacios, diversos locales que en sí son incompatibles" (Soja, 1993: 24). El espacio heterotópico es un espacio de contradicción latente. Simultáneamente, se realizan espacios diferenciales, exactamente 


\section{La actualización de la metrópolis urbana a nivel de producción del espacio, explicitando la urbanización crítica} Lenin, esclarecen la tensión dialéctica entre diferenciación e igualación, propia de las leyes del movimiento del capital, poniendo las desigualdades sociales en el centro de la explicación sobre la reproducción capitalista.

De lo abstracto a lo concreto, las visiones sobre la especialidad acababan por confluir, sintetizadas, en las concepciones de Lefebvre sobre la relación entre producción social del espacio y reproducción social de las relaciones de producción, exponiendo la necesidad de un pensamiento que incluyese la dialéctica del espacio.

El argumento de Soja (1993) sobre la geografía humana crítica lo lleva a reflexionar sobre la geografía urbana a través de las reestructuraciones urbanas, como constitutivas del proceso crítico del capital.

La economía política de la reestructuración urbana y regional, que Soja analiza a partir de Los Ángeles, demarca la dialéctica del inmueble-mueble, arreglos espaciales urbanos provisorios, o sea, pasibles de destrucciones económicas creadoras, productiva y financieramente compensadoras, propias de la economía contemporánea. El punto de ruptura de la inmovilidad involucra una red compleja de producción de lugares. La dirección de una dialéctica del espacio expone un movimiento que va de la dialéctica del tiempo a la del espacio, teniendo como intermediario, a ser negado, la fetichización del espacio; movimiento necesario para la comprensión crítica de las fuerzas estructurales del capitalismo.

\footnotetext{
9 "Al no subordinar la estructura espacial del desarrollo desigual a la clase social, sino encararla como estando 'en el mismo plano', Mandel identificó, en la escala regional e internacional, una problemática espacial que se asemeja de cerca a la interpretación de la espacialidad urbana por Lefebvre, a punto de sugerir, inclusive, una poderosa fuerza revolucionaria emergiendo de las desigualdades espaciales, que él claramente afirmó que son necesarias para la acumulación capitalista" (Soja, 1993: 103).
}

Lo que rige estas consideraciones es la tesis de que hay necesidad de una lectura de totalidad de la metrópolis para exponer su inserción en el proceso social moderno, al compás de las estrategias económicas y políticas de su mercantilización. Para esto, tres complejos analíticos sostienen el argumento, conteniendo cada uno elementos con nexos contradictorios y en estrecha conexión con los demás complejos, de manera sintética y dialéctica. A continuación se profundiza en cada uno de ellos.

\section{La crisis del trabajo, contenida en la crisis del capital}

Esta crisis se manifiesta como desempleo macizo; aumento de la composición orgánica del capital de los emprendimientos económicos, que reduce proporcionalmente el trabajo activo de la referida composición; una economía de sobrevivencia, en nuevos moldes, ya que, ante la crisis del trabajo, sectores productivos y de servicios, de baja composición orgánica del capital, son mantenidos $y$, pulverizadamente, ampliados ${ }^{10}$. Teniendo presente que la economía brasilera siempre contuvo esa presencia, las formas de dualismo que la explican son: la formalidad y la informalidad del trabajo; más recientemente, la exclusión e inclusión productiva de los trabajadores, entre otras.

\footnotetext{
10 "La tendencia a la formalización de las relaciones sociales se estancó en los años 1980, y se expandió lo que aún es impropiamente Ilamado trabajo informal [...] la desconstrucción de la relación salarial que se da en todos los niveles y sectores. Tercerización, precarización, flexibilización, desempleo a tazas de $20,6 \%$ en la Gran São Paulo -datos de $2003[\ldots]-$, y no tan contradictoriamente como se piensa, ocupación, y no más empleo [...]" (Oliveira, 2003: 142). "Entonces, gracias a la productividad del trabajo, desaparecen los tiempos de no trabajo: todo el tiempo de trabajo es tiempo de producción" (Oliveira, 2003: 136).
} 
"Todo tiempo de trabajo es tiempo de producción" coincide con el análisis de la disociación entre rendimiento (proletario) y trabajo (productivo en la fábrica). O sea: "de esta lectura se desprende, en el orden del día, la crisis del trabajo -la actual fase histórica siendo marcada por el fin del trabajo como centro de gravedad de la vida y fundamento del lazo social. Sobre el terreno de la acción colectiva, esta crítica equivale a poner una disociación entre rendimiento y trabajo [...]" (Martini-Scalzone \& Scalzone, 1990: 226227).

Dentro de esta situación, de fundamento crítico, existe el sentido de que en todo y cualquier espacio, y en todo y cualquier tiempo, se buscan formas de sobrevivencia, que incluyen, además de modos precarios de trabajo, formas de asistencia institucionalizadas (auxilio familiar, programa de renta mínima, entre otros). El programa Fome Zero es ilustrativo de la vía estructural en curso, en los países periféricos del capitalismo. Es interesante notar que las grandes metrópolis constituyen espacio-temporalmente esa presencia del trabajo en tiempo integral, completamente desvalorizado.

\section{Ciudades como lugares de producción mercantil del espacio}

Las ciudades pasan a constituir lugares privilegiados de producción mercantil del espacio, internalizando la metamorfosis del capital productivo en capital financiero. No son espacios del hombre habitante como, por algún tiempo definía Le Lannou, ni exactamente espacios del hombre productor $y$ del hombre consumidor, como evalúa Pierre George, sino espacios del hábitat y de los negocios, que niegan al hombre; espacios de deshumanización y sometimiento social e individual. De modo fenoménico, las ciudades se volvieron sujetos sociales ${ }^{11}$; en su esencia, es el proceso de urbanización, teniendo

\footnotetext{
11 Se habla de protagonismo de las ciudades: "entre los ítem de inserción: la promoción económica para el exterior y la concepción e implementación de proyectos urbanísticos" (Barreto, 2004: 12). La concepción de los proyectos urbanos tiene por base el tratamiento de la ciudad como empresa-ciudad (Osmont, 1995: 281).
}

como fundamento la proletarización absoluta. Desplazar al sujeto en la dirección del proceso de urbanización significa que todos los espacios y tiempos sociales son absorbidos, tendenciosamente, por el proceso del capital. El espacio como un todo se mueve, económicamente, según las necesidades de la economía urbana, voraz, enteramente basada en la urbanización como negocio. Bajo ese fundamento, no hay cómo identificar un sujeto, si no el inmanente a la propia economía deshumanizadora. No hay un sujeto y su hábitat, como vivienda degradada, hay el hábitat, negando al habitante, en el interior de una economía, que niega, contradictoriamente, el trabajo. Dialécticamente, aparecen como necesidad de vivienda y necesidad de trabajo. Decir que el habitar se transforma en hábitat, significa decir que el habitante no es el sujeto, sino que lo es el negocio inmobiliario, y todas sus extensiones económico-políticas. Decir que hay negación del trabajo es decir que existe una economía que se mueve, contradictoriamente, por destitución del trabajo y haciendo todo el tiempo humano un tiempo de todo y cualquier trabajo. Sujeta, no es sujeto. En el fondo, son alienaciones por destitución de apropiaciones sociales e individuales posibles. El desarrollo de las fuerzas productivas sociales, absorbidas por el capital, cada vez más financierizado, se resuelve, socialmente, inclusive, comportando restos de técnicas, insertadas en la vida cotidiana urbana. En las inmensas periferias, la de los centros históricos y expandidos de las ciudades, o la de su entorno, cada vez más amplio y distanciado, metropolitano, prevalece la baja composición orgánica del espacio, que aparece como falta de infraestructura urbana. Se designa como urbanización crítica. El movimiento es ir distanciando los espacios periféricos e ir constituyendo nuevas centralidades económicas metropolitanas ${ }^{12}$. El planeamiento espacial es parte constitutiva de las estrategias político-económicas.

El trabajo intelectual se resuelve en la realización superestructural de una pesada carga legal, de escalas multiplicadas y sobrepuestas

\footnotetext{
12 Especialmente significativo sobre este respecto, entre otros, de toda una generación de nuevos geógrafos devotos al tema es la investigación de Ana Fani Alessandri Carlos (2001).
} 
-leyes regionales, municipales y federales; combinadas con reglamentos sectoriales- en una enorme burocratización, incluyendo además del aparato estatal, organizaciones no gubernamentales, con vistas a asegurar el ennoblecimiento de los espacios degradados socialmente, incluyendo justificativos sociales, para que acabe realizándose, especialmente, como una economía urbana o producción del espacio urbano. En el interior de esta superestructura hay conquistas sociales, proporcionalmente, muy inferiores a la economía en movimiento.

La Ley No 10.257, referente al Estatuto de la Ciudad (São Paulo), reglamentada en 2001, significa, contradictoriamente, una modernización del aparato legal con vistas al derecho de propiedad. Desde el punto de vista de las operaciones urbanas, equivale a la manipulación de los índices y características de uso y ocupación del suelo y a un proceso de financierización de la propiedad privada de la tierra urbana ${ }^{13}$. Y, al mismo tiempo, instituye la función social de la propiedad, como combate a la especulación urbana, sirviendo de justificativa política a las ocupaciones urbanas, realizadas por movimientos sociales urbanos, reiterada y violentamente combatidas, a través de instituciones del Estado; reprimidas como invasiones de propiedad ajena.

Los contenidos de la necesaria dialectización del espacio involucran: el aparato legal de apoyo, sostenido por una lectura estratégica del espacio, apoyada en lógicas espaciales que rematan y sintetizan mecanismos económicos y políticos, en este caso, metropolitanos; su desdoblamiento financiero y social, ya que se establece que la economía

\footnotetext{
13 "Bajo la forma de Operaciones Urbanas, los interesados en construir grandes edificios que extrapolan la zonificación 'normal' pueden hacerlo en las regiones delimitadas por ese instituto, bastando para eso la compra de 'aditivos' a la propiedad que pueden ser adquiridos de la propia municipalidad, o de particulares, en vista de que son derechos reales, representados por papeles de libre negociación. Con la raridad del espacio horizontal se creó la raridad del espacio vertical, aunque ficticia, bajo la representación de títulos que en la ciudad de São Paulo se Ilaman CEPAC (o Certificados de Potencial Adicional de Construcción), que son igualmente finitos, aunque sean solo números" (Baitz, 2007: 95).
}

urbana va tejiendo un proceso de reproducción del capital inmobiliario, inclusive de su financierización, productor del hábitat y de los negocios, en el seno de la producción del espacio urbano, por lo tanto, definiendo formas de sometimiento social. En el caso de São Paulo, la composición o el conjunto de las Operaciones Urbanas Consorciadas, del Plano Director Estratégico del Municipio, proyectadas y existentes, se revela claramente como espacio instrumental, instruyendo los mecanismos económicos y financieros.

La movilización de la propiedad privada de la tierra, en el interior de la producción del espacio urbano, tiene un desdoblamiento de segundo grado: además de filtrar en un segmento productivo como el del capital inmobiliario, e inclusive a través de él, se reduce en su financierización y en la implicación de instituciones del Estado, directamente, en la manipulación del agenciamiento financiero ${ }^{14}$. Como proceso de urbanización, se configura un circuito propio de capital inmobiliario, con sus consecuencias tanto en el proceso del capital, como en los límites de la vida urbana; lo que se observa hoy, como inmediatamente materializado. Y ese circuito deja de ser secundario en el conjunto de los sectores económicos. Negativamente, se define una acumulación primitiva del espacio; un proceso de expropiación, combinado a la degradación del trabajo, antes anunciada.

La producción de una nueva centralidad es, inmediata y sensiblemente, la expropiación de los usos anteriores de ese mismo lugar. Se podría hablar de producción de escalas geográficas: se produce una centralidad $y$, al mismo tiempo, su periferia, incluidas en el mismo proceso (Smith, 2002).

En el caso de São Paulo, las estrategias inmobiliarias renovadas sugieren la dirección suroeste, preferentemente. Estrategias que se expanden, también por otros ejes de

\footnotetext{
${ }^{14}$ Obsérvense los CEPAC, instrumentos financieros conectados a las Operaciones Urbanas, convertidos en títulos, alienados en remates, vendidos en el mercado, pudiendo ser, de modo ficticio, valorizados. Lo que se pretendía era recaudar fondos para las inversiones definidas como públicas, pero, en realidad, se juegan las inversiones, en conjunto con el sector privado.
} 
valorización, lo que lleva al Rodoanel (gran anillo viario metropolitano perimetral), como la frontera diseñada de los nuevos negocios potenciales de la urbanización.

Bajo el punto de vista de la urbanización crítica, es posible examinar la producción del espacio en un proceso de continuidaddiscontinuidad de la producción industrial en la moderna sociedad capitalista: determinados sectores productivos adquieren potencia entre los negocios -las industrias de la construcción, que dejan de ser un sector secundario-; se desarrolla un sistema complejo de comercialización del producto-espacio -las incorporadoras e inmobiliarias internacionalizadas y consorciadas en lo urbano a punto de producir territorios de negocios-; la circulación de la riqueza producida y ficticia involucra un proceso ampliado de financierización; políticas diversas amparan la pulsación de las necesidades de esa producción y la producción del espacio actualiza la importancia de la movilización de la moderna propiedad de la tierra -aquí también se va de extracción de rentas de la tierra urbana, a partir de estrategias económicas y urbanas, a rentismos varios, en la orilla de tales estrategias de negocios urbanos. Se podría hablar de una estratificación espacial de las centralidades: de centralidades locales, que facilitan la vida cotidiana de la población en su movilidad diaria y ofrecen los servicios y comercios básicos; a las regionales (en la misma zona de la metrópolis), que incluyen las redes comerciales y de servicios de mayor porte; a las metropolitanas, cuyo acceso es más reducido, y que pueden incluir una inserción profesional necesaria a la sobrevivencia; y así sucesivamente.

Por ese carácter, el de la densidad relativa de composición orgánica del espacio, como momento necesario de elucidar la urbanización crítica, se insiste en la dialéctica centro-periferia ${ }^{15}$. Al mismo tiempo se tiene la producción de nuevas centralidades, que

\footnotetext{
15 "La noción de centro y de periferia es una noción muy importante en la condición de ser precisada y diferenciada". Sugiere "siempre nuevas centralidades y nuevas periferias". Es posible considerar la noción de centralidades subordinadas (Renaudie, 1988: 62-66).
}

conducen a la movilidad espacial de la población originaria del lugar y al empuje para la frontera de esa nueva nucleación.

\section{Destitución de la humanidad como naturalización de lo humano}

La profunda destitución de la humanidad del hombre es, al mismo tiempo, la naturalización de lo humano. Martini-Scalzone \& Scalzone (1990) dicen "La crítica ecológica comprendió en la raíz una de las cuestiones centrales de nuestra época: la relación entre modernización y catástrofe" (MartiniScalzone \& Scalzone, 1990: 228). Hay una exaltación de la naturaleza y de lo natural, llenando el vacío de proyectos políticos de superación de innumerables crisis sociales. La potencia ideológica de lo ambiental, sustituyendo el caos socioespacial es extremadamente paradójica, porque, en São Paulo, ha llegado a ser justificativo para el Rodoanel Mário Covas, un proyecto sectorial del área de los transportes, definiendo un sistema viario perimetral, que circunda la metrópoli de São Paulo a la altura de lo designado como anillo periurbano; dividido en cuatro tramos, el trecho oeste ya está en funcionamiento (desde 2002) y el trecho sur está en producción. En esos dos trechos, ya son $90 \mathrm{~km}$ de carreteras previstas. Faltan los trechos norte y este, que cerrarían el circuito de alrededor de $175 \mathrm{~km}$, a una distancia de 20 a $40 \mathrm{~km}$ del centro histórico de São Paulo. El trecho sur ya precipita una conexión posible con lo que se constituirá en el trecho este, y así sucesivamente. Nuevamente prevalece una noción de conjunto del área metropolitana a irrigar, económicamente.

Para justificar una carretera así de extensa y su sentido, en la base de una nueva plataforma logística para los negocios metropolitanos, se induce al Ilamado efecto barrera, inhibidor de la ocupación irregular (Governo do Estado de São Paulo, 2004) y del nuevo avance de lo que fue Ilamado urbanización desordenada ${ }^{16}$-ocupaciones irregulares, desde el punto de vista de la propiedad

\footnotetext{
16 El concepto de urbanización desordenada, que induce a la idea de planificación, contradice al de urbanización crítica.
} 
privada de la tierra y urbanístico: favelas y loteos clandestinos en su mayoría-; denota lo que se definió como anillo periurbano. Es exactamente el sistema de carreteras en implantación lo que se presenta como un elemento propulsor de la calidad ambiental de espacios metropolitanos; estos ya definidos como áreas de conservación y protección ambiental (especialmente en las zonas sur, suroeste y norte de la metrópoli), con una legislación datada de los años 1970, que fue sucumbiendo a los sucesivos fracasos de los avances de la urbanización metropolitana y, repetidamente, reformulada.

Las leyes de protección ambiental indican la magnitud de la periferia urbana involucrada y por eso sufren innumerables modificaciones, absorbiendo y revelando, a través de esas modificaciones, las propias dificultades de su realización. Aún más, ellas mismas justifican el crecimiento del mercado popular de tierras en ciertas áreas protegidas, que son, con esas leyes, desvalorizadas para otros usos; como es el caso de gran parte de la zona sur de São Paulo ${ }^{17}$.

El motor económico de ennoblecimiento de esos espacios periurbanos, a través de nuevos condominios, proyectos económicos y actualizaciones de infraestructura urbana, se combina con la ideología de la preservación y conservación ambiental. Se retira el inconveniente de millones de personas, allí fijados, restando la combinación entre naturaleza y economía. La primera como base estructural; la segunda, su superestructura ideológica de soporte. Aunque, paradójicamente, justificando una carretera expresa y sus varias extensiones.

El espacio instrumental, así conceptualizado, por Henri Lefebvre, en la metrópoli de São Paulo, sirve para configurar una sustitución crucial: la neutralización de lo social por medio de la naturaleza-natural.

\footnotetext{
17 A propósito de los loteos de la represa Guarapiranga, investigaciones recientes sugieren un mercado informal de tierras muy lucrativo, impulsado por la propia legislación ambiental. Lo mismo se repite en la Billings.
}

El Rodoanel Mário Covas, como sistema de rutas perimetral, proyectado y en ejecución desde los años 1990, atraviesa la metrópoli de São Paulo ${ }^{18}$ y demarca el círculo y circuito de realización y renovación potenciales de valorización económica de esta metrópoli ${ }^{19}$. Ofrece la imagen del perímetro urbano metropolitano que fue diseñado y se está implementando, formando un gran anillo de valorización económica del espacio. Determina un contexto y una imagen espaciales de la totalidad sobre la producción del espacio urbano e, inmediatamente, impulsa un proceso de expropiación social, propio de la acumulación primitiva del espacio, que forja el espacio de valorización.

Una noción de conjunto de la realidad urbana economizada se hace más patente si se Ilena esa imagen totalizante del Rodoanel Mário Covas con otros proyectos, como los de las Operaciones Urbanas en São Paulo.

18 El proyecto Rodoanel Mário Covas se refiere a un anillo vial metropolitano cerrado, cortando las principales carreteras de acceso a la metrópoli, cuya producción es controlada por el gobierno del Estado de São Paulo y por el gobierno federal. El primer trecho, oeste, interconecta Régis Bittencourt con la carretera vieja de Campinas, en Perus, pasando por las carretera Anhanguera, Bandeirantes, Castelo Branco y Raposo Tavares, con 32 km, este está terminado. El segundo trecho, sur, va a conectar la carretera Régis Bittencourt con la Anchieta (pasando por la carretera Imigrantes), teniendo alrededor de $40 \mathrm{~km}$. Completan el circuito los trechos norte y este. El anillo vial metropolitano involucra, además de São Paulo, 15 municipios más. En el trecho oeste, están Barueri, Carapicuíba, Cotia, Embu e Osasco. En el sur, Embu, Itapecerica da Serra y São Bernardo do Campo. En el este, Mauá, Ribeirão Pires, São Bernardo, Ferraz de Vasconcelos, Itaquaquecetuba, Guarulhos, Poá y Suzano. En el norte, Guarulhos.

${ }^{19}$ Hay una metamorfosis de la funcionalidad de la presencia del trabajador potencial en la del morador temporal. La valorización y capitalización de las periferias, inclusive reproduciendo formas de especulación financiera, constitutivas de centralidades potenciales, terminan por llevar a una acumulación primitiva de esos espacios; o sea, una eliminación de sus usos y moradores existentes, en pro de nuevas estrategias y emprendimientos. Considerando la inmensidad de las periferias, las estrategias de expropiación deben ser gigantescas, a ejemplo del Rodoanel Mário Covas, en São Paulo, que involucra, potencialmente, la metrópoli entera. 
El programa Rodoanel Mário Covas puede estar definiendo la magnitud necesaria de las políticas urbanas, con vistas a movilizar económicamente las inmensas periferias metropolitanas. Tal programa se presenta como política económica sostenible, corrigiendo la pésima calidad ambiental de las áreas periféricas pobres.

La urbanización, definida crítica, cuyo fundamento subjetivo negado es la masa proletaria de las periferias metropolitanas, sujeta a los avances del capital inmobiliario, y de otras formas de capital, financierizadas, y bajo el incentivo estadista, incluye la producción de representación en télescopage ${ }^{20}$, entre la crisis social y la crisis ambiental, a partir de políticas de sostenibilidad, como aquello que encierra la imagen ilusoria de equilibrio.

La manera como la imposibilidad de lo urbano aparece plantea desequilibrios constitutivos: en los conjuntos habitacionales y en otras formas de loteo urbanos, las densificaciones, que comprometen la urbanización de los mismos; el control de los que podrán ser parte de actividades de ayuda mutua para hacerlos viables; el recelo de las invasiones, en una ocupación; el choque entre las cuestiones sociales y las específicamente ambientales, hacer el lugar más urbano significa, en los límites de esta reproducción social crítica, expulsar a la población que es excedente; y dependiendo de sus términos, esta selección es casi neutralizada.

¿Es posible permanecer en la télescopage, de los problemas ambientales y sociales?, ¿sería posible administrarlos?, ¿sería posible neutralizar la centralidad de lo económico en la sociedad moderna?

Es posible comprender la revisión económica del territorio metropolitano urbano, actualizando la metrópoli de São Paulo, para los nuevos negocios urbanos. La misma debe recibir una macrodimensión para mover un

\footnotetext{
${ }^{20}$ El término fue configurado por Henri Lefebvre (2000). La télescopage está en el plano de la producción de una ilusión, de una confusión, de una mezcla de realidad y representación, potencializado, por transferencia y redefinición de contenidos, terriblemente activas.
}

área extensa de usos residenciales populares y dar lugar a nuevas inversiones. Las polémicas, entre los últimos alcaldes, a partir de finales de los años 1980, demuestran cómo esas nuevas estrategias estatistas, configurando el territorio economizado de la metrópoli, aún están en curso y definición.

\section{Consideraciones finales}

Algunos elementos, de lo que fue tratado, intentan ser una representación analítica de lo urbano economizado como totalización, y de los restos que sobran a la masa proletaria. No son hechos, son procesos; están en curso y se realizarán más o menos, en el tiempo y en el espacio, según la eficacia de las formas de actualización de la metrópoli de São Paulo, en la tentativa de inserción en la circulación global de los negocios que mueven el mundo. Y depende del lugar en el mundo economizado, de esa porción que es la metrópoli de São Paulo. Es una escala producida y en producción, que depende de las otras, internacionalizadas, para realizarse de hecho. Se diría, está en preparación el terreno para esa posibilidad, en los términos de la producción social del espacio, y la extensión de la crisis social, que precipita.

\section{Referencias bibliográficas}

BAITZ, R. A propriedade contra a posse e a propriedade 2. In: GEOUSP. Espaço e tempo. São Paulo: Departamento de Geografia, FFLCH, USP, 2007, p. 91-109.

BARRETO, M. I. Inserção internacional de governos locais. Revista Teoria e Debate, 2004, No 59, p. 12-16.

CAPEL, H. Filosofía y ciencia en la geografía contemporánea. Barcelona: Barcanova, 1981.

CARLOS, A. F. A. Espaço-tempo na metrópole. São Paulo: Contexto, 2001.

GEORGE, P. Geografia urbana. São Paulo: Difel, 1983.

GEORGE, P. La Ville-Le fait urbain a travers le monde. París: Presses Universitaires de France, 1952. 
GEORGE, P.; GUGLIEMO, R.; LACOSTE, Y. \& KAYSER, B. A geografia ativa. São Paulo: Difusão Européia do Livro/EDUSP, 1966.

GOVERNO DO ESTADO DE SÃO PAULO. Avaliação ambiental estratégica do programa Rodoanel. São Paulo: Governo do Estado de São Paulo, 2004.

HARVEY, D. A justiça social e a cidade. São Paulo: Hucitec, 1980.

HEGEL, M. C. Propedêutica filosófica. Paris: Gallimard, 1995.

JOHNSTON, R. J. Geografia e geógrafos. São Paulo: Difel, 1986.

LEFEBVRE, H. Descartes. Paris: Éditions Hier et Aujourd'hui, 1947.

LEFEBVRE, H. Le droit à la ville suivi de Espace et politique. París: Anthropos, 1974.

LEFEBVRE, H. La révolution urbaine. París: Gallimard, 1970.

LEFEBVRE, H. Production de I'espace. París: Anthropos, 2000.

MARTINI-SCALZONE, L. \& SCALZONE, O. Écologisme et autonomie. IN: GROUPE
DE NAVARRENX. Du Contrat de Citoyenneté. París: Syllepse/Périscope, 1990, p. 221 246.

MOSCONI, P. (org.). Internationale Situationniste. París: Fayard, 1997.

OLIVEIRA, F. Crítica a la razón dualista/ O ornitorrinco. São Paulo: Boitempo, 2003.

OSEKI, J. H. O único e o homogêneo na produção do espaço. In: MARTINS, J. (org.). Henri Lefebvre e o retorno à dialética. São Paulo: Hucitec, 1996, p. 109-119.

OSMONT, A. La banque mondiale et les villes-du développement à I'ajustement. París: Karthalam 1995.

RENAUDIE, S.; RÉGULIER, C.; MULLER, A. \& LEFEBVRE, H. Henri Lefebvre-uma nova positividade do urbano. Journal M, 1988, p. 62-66.

SMITH, N. Geografía, diferencia y políticas de escala. Terra Livre, 2002, No 19, p. 127-145.

SOJA, E. Geografias pós-modernas-a reafirmação do espaço na teoria social crítica. Rio de Janeiro: Jorge Zahar, 1993. 